\title{
State Forecast Method of Electronic Equipments Based on Improved HMM and LS-SVM
}

\author{
Jianzhong Zhao \\ Department of ordnance science and technology \\ Naval aeronautical engineering university \\ Yantai, China \\ zjznavy@163.com
}

Wen Ye

Department of ordnance science and technology Naval aeronautical engineering university

Yantai, China

Yewen79@163.com

\author{
Yong Liu \\ Group of receiving and improving equipment \\ Naval aeronautical engineering university \\ Yantai, China \\ Liuyong1973@163.com \\ Tao Jiang \\ Department of ordnance science and technology \\ Naval aeronautical engineering university \\ Yantai, China \\ ktyl@163.com
}

\begin{abstract}
For the deficiency that the traditional single forecast method can not forecast precisely the states of electronic equipments, a combined forecast method based on hidden Markov model (HMM) and least square support vector machine (LS-SVM) is presented. Multi-agent genetic algorithm (MAGA) is used to estimate parameters of HMM in order to overcome the problem that Baum-Welch algorithm is easy to fall into local optimal solution easily. MAGA is used to estimate parameters of LS-SVM. On the base of these, the combined forecast model of electronic equipment states is established. The example results showed the superiority of the combined forecast model at forecast precision, calculation speed and stability.
\end{abstract}

Keywords-parameter estimation; hidden markov model(HMM); least square support vector machine(LS-SVM); multi-agent genetic algorithm (MAGA); state forecast

\section{INTRODUCTION}

States forecast methods are essential science and technology. Least square support vector machine (LSSVM) and Hidden Markov model (HMM) are the common forecast methods ${ }^{[1,2]}$. At present, the documents about research on combining HMM and SVM are less comparatively, that focus on mainly voice distinguishing, image objective and fault diagnosis ${ }^{[3,4]}$. The research results show the excellent performance of the combined method. In these study HMM is used in classification implement but massive train calculation and long-time calculation are needed. HMM has the decoding function when single HMM is trained. In the paper HMM was combined with LS-SVM as decoder. LS-SVM was used to forecast the observation figure of equipment state and HMM was used to solve the future state of equipment, that made fault forecast be relevant with equipment state directly. Under these circumstances forecast results were easily understood and analyzed, that could offer decision reference for maintaining support person.

\section{THE IMPROVEMENT OF HMM}

HMM is a double random process ${ }^{[5]}$ : one random process is the Markov chain describing finitude state of state transformation, the other random process is corresponding relation of statistics between every state and observation figure.

The solution gotten by Baum-Welch ( B-W ) algorithm is not certain that these are entirely optimal resolution overall situation, but is usually one only local optimal resolution ${ }^{[6]}$. For the problem of HMM, the algorithm was improved by multi-agent genetic algorithm ( MAGA) in the paper. MAGA is a new optimization method based on the ability of intelligence agent apperceiving environment and reaction, main features of that are small scale population, fast convergence, better seek ability overall situation and stability ${ }^{[7,8]}$.

For electronic equipment performance degeneration can not usually be duplicated, thus left-right and right-turn model is taken as HMM model, that is illustrated by the first figure. The state of one is initial state, the state of five is fault state, and the state of two, three, and four are different degree degeneration state.

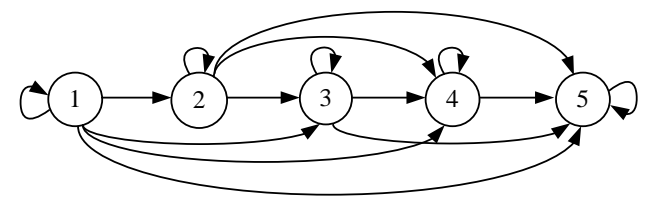

Figure 1. HMM states transfer chart of electronic equipments

1) Chromosome code. The code way of real number is adopted, that is illustrated by the second figure. Every chromosome is composed of $\pi$ area, A area and B area, 
that is also three parameter of HMM model. State transformation probability matrix is under the condition of $a_{i j} \neq 0$,only $j \geq i$, and $a_{N N}=1$. Therefore the genes of A area is the element of matrix A that is not zero, in that $a_{N N}$ is not included.

$$
\begin{aligned}
& \left\{\begin{array}{cc}
\sum_{i} \pi_{i}=1, & i=1,2, \cdots, N \\
\sum_{j} a_{i j}=1, & i=1,2, \cdots, N-1 ; j=i, \cdots, N \\
\sum_{k} b_{i k}=1, & i=1,2, \cdots, N ; k=1,2, \cdots, M
\end{array}\right.
\end{aligned}
$$

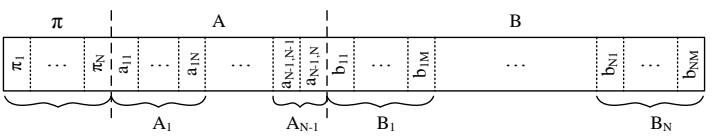

Figure 2. Chromosome coding of parameters estimation

$L_{i, j}$ is regarded as chromosome, then $L_{i, j}$ is one real figure vector:

$$
L_{i, j}=\left(\pi_{1}, \cdots, \pi_{N}, a_{11}, \cdots, a_{N-1 N}, b_{11}, \cdots, b_{N M}\right)
$$

2) Operation achievement way of genetic operator. The genetic operator of MAGA is mainly consist of neighbourhood competition operator, neighbourhood crossover operator, variation operator and self-learning operator Self-learning of intelligence agent, while selflearning operator included the front three operator. In order to ensure the frist formula exist throughout, the front three operator is not operated exceeding own area, but is operated alone in proper order in respective area. These operation is carried out inside one section or among sections, every of that is normalization disposed.

3) Fitness function. The goal of HMM parameter estimation is to seek parameter $\lambda=(\boldsymbol{\pi}, \boldsymbol{A}, \boldsymbol{B})$ so as to make $P(\boldsymbol{O} \mid \lambda)$ reach ${ }^{\text {maximal }}$ value, thus $P(\boldsymbol{O} \mid \lambda)$ resolved by front direction algorithm is regarded as adaptation degree function. But considering $P(\boldsymbol{O} \mid \lambda)$ is less than 1 , the logarithm of $P(\boldsymbol{O} \mid \lambda)$ is regarded as adaptation degree function so as to compare adaptation degree. Supposed the number of observation array regarded as train is $K$, the observation probability of the $k$ observation array is $P\left(\boldsymbol{O}^{(k)} \mid \lambda\right)$, thus adaptation degree function is:

$$
f_{f i t}(\lambda)=\ln \left[\frac{1}{K} \sum_{k=1}^{K} P\left(\boldsymbol{O}^{(k)} \mid \lambda\right)\right]
$$

4) Evolution end condition. Because the specific figure of $P\left(\boldsymbol{O}^{(k)} \mid \lambda\right)$ is undefined, the method making evolution end by adaptation figure threshold is not applicative. Therefore maximum evolution algebra is regarded as end condition in the paper.

\section{THE IMPROVEMENT OF LS-SVM}

For LS-SVM, optimization indexes of LS-SVM are indicated by square term, inequality restraint is replaced by equation restraint, the twice programming problem is transformed into linear equation group so as to solve, that simplifies solving process ${ }^{99]}$.
The optimization estimation on nucleus parameter $\sigma$ and punishment parameter C of LS-SVM is actually a complex optimization problem of up-down limit continuous function, thus that is estimated by MAGA in the paper.

1) Chromosome code. Parameter figure range of LSSVM is large. If binary system coding is adopted, the chromosome length could not reach precision demand if it is too short, on the other hand the chromosome length is likely to increase calculation complexity degree of coding again and again if it is too long. Real number coding way is adopted in the paper, that is illustrated by the fourth figure.

2) Genetic operation operator. Neighborhood competition operator is iterated according to the way that intelligence agent with maximum energy in its neighborhood. The crossover position is not selected when neighborhood crossover operator crossed, a part of nucleus parameter $\sigma$ of intelligence agent is exchanged partially, and the cross operation occurred only once. Variation operator needed some improvement. Variation occurred according to the relation among the intelligence agent energy impacted by variation operator, maximum energy of intelligence agent and population average energy. Finally the original intelligence agent is replaced by the new intelligence agent that had maximum energy. Selflearning operator impacted usually on the intelligence agent that had maximum energy, of that population initialization problem needed to be solved.

3) Adaptation degree function. In order to validate which is parameter estimation the excellent or not, root mean square error (RMSE) that is trained by cross validation method is regarded as adaptation degree function. The form of adaptation degree function is illustrated by the fourth formula:

$$
f_{\text {fit }}(\sigma, C)=\sqrt{\frac{1}{N} \sum_{i=1}^{N}\left(y_{i}-\hat{y}_{i}\right)^{2}}
$$

4) End condition. In order to guarantee appropriate arithmetic quantity, two end condition is used that is maximum evolution algebra and minimum RMSE. Maximum evolution algebra is set for 100, minimum RMSE is set for 0.02 . If one of the two end condition happened, the algorithm ended.

\section{COMBINED FORECAST MODEL BEING ESTABLISHED}

The degenerating state of electron equipment is not visible, but is expressed out by the performance parameters that could be observed, thus degenerating state of electron equipment is very difficult to be forecasted directly by LSSVM. The possible state of equipment could be estimated by the way of observation value when HMM is used as decoder. Therefore LS-SVM and HMM could be combined so that degenerating state of electron equipment is forecasted directly. First the performance date (possible observation date in future) of equipment is forecasted, then the degenerating state of equipment is estimated according to forecast date by HMM. The flow of combined forecast model is illustrated by the third figure. 


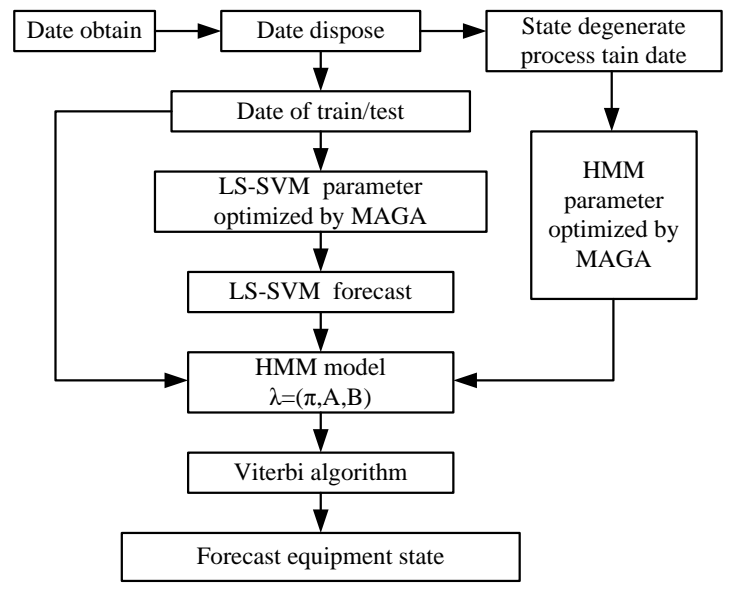

Figure 3. The flow chart of combined forecast method

1) Parameters train is carried out according to degenerating process date of electron equipment (observation date) that had be disposed;

2) Regression parameters of LS-SVM is estimated by MAGA.

3) Performance parameters of electron equipment is forecasted nonlinearity by LS-SVM trained so as to get forecast observation value;

4) Forecast observation value and previous observation date is imported into HMM, then future degenerating state of equipment is estimated by Viterbi algorithm ${ }^{[10]}$. Electron equipment state is divided into five grades: "health", "fine", "notice", "deterioration", "bad".

\section{EXAMPLE ANALYSIS}

Temperature control amplifier is a complex electron product and a very important accessory on the a great many of electromechanical equipment. The temperature of electromechanical equipment pivotal position could be controlled and air flow and fuel oil flow could also be rectified by temperature control amplifier. But it is the especial component that usually damaged because of installment position and work surroundings, thus it is very essential to be carry out state forecast for temperature control amplifier.

\section{A. Date disposing}

Twenty temperature control amplifier was selected as research object. The four parameter of T1, T3, T6 temperature signal voltage and speed-decreasing warning voltage was simulation date, but date was not listed because of limited article length.

The initial normal state date of the parameters about $\mathrm{i}$ equipment was $x_{i 0}^{p}(\mathrm{p}=1, \ldots, 4 ; \mathrm{i}=1, \ldots, 20)$, degenerating process date was $x_{i k}^{p}(\mathrm{k}=1,2, \ldots)$, then $\left|x_{i k}^{p}-x_{i 0}^{p}\right|$ is absolute change volume of parameter $\mathrm{p}$ at test moment $\mathrm{k}$, and that

$$
\sum_{p}\left|x_{i k}^{p}-x_{i 0}^{p}\right|
$$

The sum of absolute change volume of four parameters was at test moment $\mathrm{k}$. The fusion dates gotten form the fifth formula were used as simulation calculation date.

\section{B. Parameter estimation}

Parameter set of MAGA that was used to parameter estimation for HMM and LS-SVM was illustrated by the frist table. According to the different degree degenerating of temperature control amplifier, the number of degenerating state was set as five, thus the state number of HMM was $\mathrm{N}=5$, and the number observation value corresponding with every state was $\mathrm{M}=5$. Minimum RMSE was supposed as 0.02, and the threshold of LSSVM was $\alpha_{\text {min }}=0.001$.

TABLE I. PARAMETERS SETTING OF MAGA

\begin{tabular}{|c|c|c|c|c|c|}
\hline \multicolumn{3}{|c|}{ MAGA parameter } & \multicolumn{3}{c|}{ Self-learning operator parameter } \\
\hline & HMM & LS-SVM & & HMM & LS-SVM \\
\hline$L_{\text {size }}$ & 8 & 7 & $s L_{\text {size }}$ & 3 & 3 \\
\hline$P_{o}$ & 0.15 & 0.15 & $s P_{r}$ & 0.15 & 0.15 \\
\hline$P_{c}$ & 0.7 & 0.7 & $s P_{c}$ & 0.85 & 0.75 \\
\hline$P_{m}$ & 0.15 & 0.1 & $s P_{m}$ & 0.05 & 0.05 \\
\hline Gen & 200 & 100 & $s G e n$ & 10 & 5 \\
\hline
\end{tabular}

Parameter figures of HMM was gotten by MAGA optimization after five operation. State transfer probability matrix was:

$$
\boldsymbol{A}=\left[\begin{array}{ccccc}
0.7921 & 0.1079 & 0.0653 & 0.0204 & 0.0143 \\
0 & 0.7132 & 0.1365 & 0.0648 & 0.0855 \\
0 & 0 & 0.6614 & 0.2897 & 0.0489 \\
0 & 0 & 0 & 0.7946 & 0.2054 \\
0 & 0 & 0 & 0 & 1
\end{array}\right]
$$

Observation probability matrix was:

$$
\boldsymbol{B}=\left[\begin{array}{lllll}
0.3179 & 0.2091 & 0.1901 & 0.2324 & 0.0505 \\
0.2819 & 0.3127 & 0.2073 & 0.1146 & 0.0835 \\
0.1854 & 0.2219 & 0.2632 & 0.2197 & 0.1098 \\
0.1834 & 0.1662 & 0.2472 & 0.2761 & 0.1271 \\
0.2123 & 0.1937 & 0.1758 & 0.2528 & 0.1654
\end{array}\right]
$$

Initial state probability vector was:

$$
\boldsymbol{\pi}^{0}=[0.9928,0.0034,0.0026,0.0009,0.0003]
$$

The estimation results of LS-SVM parameters were gotten after five operation, that was illustrated by the second table. The nucleus parameter of $\sigma=0.4517$ and punishment parameter of $C=138.6274$ were selected.

TABLE II. THE PARAMETER ESTIMATION RESULTS OF LS-SVM

\begin{tabular}{|c|c|c|c|c|}
\hline Sequence & $\boldsymbol{\sigma}$ & $\boldsymbol{C}$ & RMSE & Algebra \\
\hline 1 & 0.4583 & 136.2593 & 0.0233 & 93 \\
\hline 2 & 0.4517 & 138.6274 & 0.0191 & 97 \\
\hline 3 & 0.4661 & 137.0518 & 0.0274 & 90 \\
\hline 4 & 0.4523 & 138.5116 & 0.0198 & 95 \\
\hline 5 & 0.4603 & 139.8352 & 0.0289 & 91 \\
\hline
\end{tabular}

In order to test the validity of MAGA further, MAGA algorithm was compared with Baum-Welch algorithm and ant colony algorithm(ACA), the result was illustrated by the third table and the fourth table, the optimization ability of MAGA was best in three algorithm. Performance of LSSVM was evaluated by three evaluation indexes of mean absolute error (MAE), RMSE and mean relative error(MRE). The fourth figure and the fifth figure was 
respectively the adaptation value curve corresponding with MAGA optimization HMM and LS-SVM parameter estimation optimal parameter, and quick convergence of MAGA could be found out from these figure.

TABLE III. RESULTS CONTRAST OF PARAMETERS ESTIMATION

\begin{tabular}{|c|c|c|c|}
\hline \multirow{2}{*}{$\begin{array}{c}\text { Operation } \\
\text { number }\end{array}$} & \multicolumn{3}{|c|}{$\begin{array}{c}\text { The biggest logarithmic average likelihood } \\
\text { probability value }\end{array}$} \\
\cline { 2 - 4 } & MAGA & Baum-Welch & ACA \\
\hline 1 & -4.4153 & -21.5773 & -8.7419 \\
\hline 2 & -4.3677 & -15.1851 & -6.3924 \\
\hline 3 & -4.3869 & -10.2419 & -9.6077 \\
\hline 4 & -4.4275 & -18.9137 & -9.1859 \\
\hline 5 & -4.3818 & -26.6025 & -10.2467 \\
\hline
\end{tabular}

TABLE IV. CONTRAST OF PARAMETER ESTIMATION PERFORMANCE OF THREE ALGORITHMS

\begin{tabular}{|c|c|c|c|}
\hline & AGA & ACA & MAGA \\
\hline MAE & 0.0298 & 0.0253 & 0.0147 \\
\hline MAE & 0.0439 & 0.0335 & 0.0167 \\
\hline RMSE & 0.0393 & 0.0305 & 0.0191 \\
\hline RMSE & 0.0558 & 0.0413 & 0.0207 \\
\hline MRE & 1.0083 & 0.6915 & 0.3796 \\
\hline MRE & 1.1758 & 0.7813 & 0.4097 \\
\hline
\end{tabular}

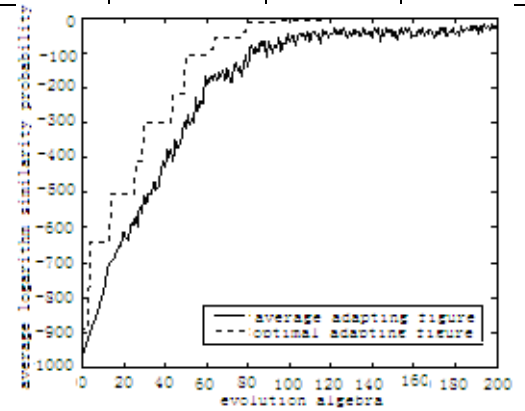

Figure 4. Fitness curve of HMM parameters optimi zied by MAGA

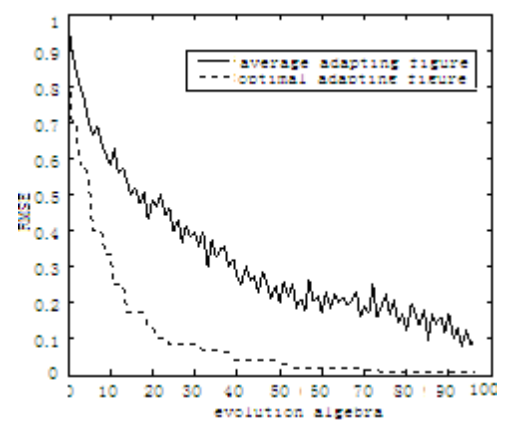

Figure 5. Fitness curve of LS-SVM parameters optimi zied by MAGA

\section{State forecast}

Ten feature fusion dates of one temperature control amplifier were selected as test dates of LS-SVM according to degenerating time list, forecast result was illustrated by the sixth figure. It was necessary that the forecast performance of LS-SVM optimized by MAGA was optimal, but forecast precision decreased with forecast steps increasing. The performance compare date of LSSVM optimized by three algorithms was illustrated by the fifth table.

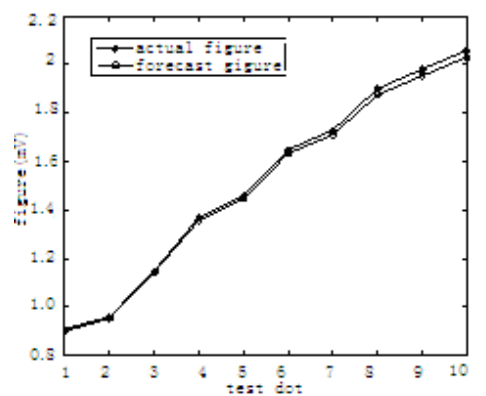

Figure 6. The actual value and the predictive value by LS-SVM

TABLE V. CONTRAST OF PREDICTION PERFORMANCE OF LS-SVM

\begin{tabular}{|c|c|c|c|}
\hline Evaluating index & AGA & ACA & MAGA \\
\hline MAE & 0.0439 & 0.0317 & 0.0203 \\
\hline RMSE & 0.0503 & 0.0384 & 0.0261 \\
\hline MRE & 1.1882 & 1.0327 & 0.5429 \\
\hline
\end{tabular}

\section{CONCLUSIONS}

HMM and LS-SVM was trained by MAGA in order to increase parameter estimation performance greatly. For the equipment with degenerating process date, the combined forecast model has favorable application prospect. On the base of state forecast, it is essential for the problem of state estimation and remaining life forecast to be researched further in future.

\section{REFERENCES}

[1] SUYKENS J A K, VANDEWALLE J, DE MOOR B. Optimal control by least squares support vector machines[J].IEEE Transactions on Neural Networks, 2001,14(1):23-35.

[2] JIANG Hua, LI Xiao-wei, LIU Cu-jun. Large margin hidden Markov models for speech recognition[J].IEEE Transactions on Audio, Speech and Language Processing, 2006,9(5):1584-1594.

[3] HUAN Rui-he, YANG Ren-leng,YUE Jin. Synthetic aperture radar images target recognition combined SVM with HMM[J]. Systems Engineering and Electronics, 2008, 30(3): 447-451.

[4] LIU Xin-meng, QIU Jia, LIU Guo-jing. HMM-SVM Based Mixed Diagnostic Model and Its Application[J]. Acta Aeronautica et Astronautica Sinica, 2005, 26(4): 496- 500.

[5] HE Xue, LI Ding. A new look at discriminative training for hidden Markov models[J]. Pattern Recognition Letters, 2007, 28(5): 12851294.

[6] JIAO L C, BO L F. Fast sparse approximation for least squares support vector machine [J]. IEEE Trans. on Neural Networks, 2007, 18(3): 685-697.

[7] VAPNIK V N. Essence of statistics study theory[M].Zhang Xuegong, translation. Beijing: Tsinghua University press, 2000:47124.

[8] DENG Ni-yao, TIAN Yue-jun. Support vector machines-theory, algorithm and expand[M].Beijing: Science press, 2009: 174-176.

[9] RABINER L R, HUANG B H. An introduction to hidden markov models[J]. IEEE Signal Processing Magazine, 1986, 3(1): 4-16.

[10] LIU Yue, NI Piao-ting, SHANG Yi-sheng. Research on failure prediction for switch power based on HMM[J]. Computer Measurement \& Control, 2011, 19(10): 44-51. 